Pacific Journal of Mathematics

A DISCONJUGACY CRITERION FOR HIGHER ORDER
LINEAR VECTOR DIFFERENTIAL EQUATIONS 


\title{
A DISCONJUGACY CRITERION FOR HIGHER ORDER LINEAR VECTOR DIFFERENTIAL EQUATIONS
}

\author{
WILLIAM T. REID
}

\begin{abstract}
For a higher order linear quasi-differential equation which is non-self-adjoint there is presented a disconjugacy criterion that is a consequence of the disconjugacy of an associated self-adjoint quasi-differential equation. In particular, there is considered the specific form of this criterion for a higher order differential equation of the canonical form which has been presented by the author, Transactions of the American Mathematical Society, 85 (1957), 446-461.
\end{abstract}

1. Introduction. For self-adjoint Hamiltonian differential systems which satisfy a condition of definiteness that in the case of accessory systems for variational problems is the strengthened Legendre or Clebsch condition, it is well-known, (see, for example, Bliss [1, Secs. 89, 90], Morse [5; 6, Ch. IV], Reid [7; 9; 11, Sec. VII. 5]), that the condition of disconjugacy is equivalent to the positive definiteness of the associated (Dirichlet) hermitian functional. In turn, for nonself-adjoint differential systems one may derive a sufficient condition for disconjugacy as a consequence of the disconjugacy of certain associated self-adjoint systems. An example of this procedure involving a linear homogeneous vector differential equation of the second order is given in Reid [7, Sec. 5]; see also, Hartman and Wintner [3]. The purpose of the present paper is to present corresponding results for more sophisticated differential systems of higher order.

Matrix notation is used throughout; in particular, one column matrices are called vectors. The $n \times n$ identity matrix is denoted by $E_{n}$, or merely by $E$ when there is no ambiguity, and 0 is used indiscriminately for the zero matrix of any dimensions. The conjugate transpose of a matrix $M$ is denoted by $M^{*}$. The symbols $M \geqq N$, $\{M>N\}$, are used to signify that $M$ and $N$ are hermitian matrices of the same dimensions and $M-N$ is a nonnegative, \{positive\}, definite matrix. A matrix function is termed continuous, integrable, etc., when each element of the matrix possesses the specified property.

If a matrix function $M(t)$ is a.c., (absolutely continuous), on a compact interval $[a, b]$, then $M^{\prime}(t)$ signifies the matrix of derivatives at values where these derivatives exist, and zero elsewhere. Similarly, if $M(t)$ is (Lebesgue) integrable on $[a, b]$, then $\int_{a}^{b} M(t) d t$ denotes the matrix of integrals of respective elements of $M(t)$. For a given interval $[a, b]$, the symbols $\mathfrak{夭}_{p q}[a, b], \mathfrak{C}_{p q}^{n}[a, b], \mathfrak{R}_{p q}[a, b], \mathfrak{R}_{p q}^{k}[a, b], \mathfrak{R}_{p q}^{\infty}[a, b]$, $\mathfrak{U}_{p q}[a, b], \mathfrak{N}_{p q}^{n}[a, b]$ are used to denote the class of $p \times q$ matrix functions 
$M(t)=\left[M_{\alpha \beta}(t)\right],(\alpha=1, \cdots, p ; \beta=1, \cdots, q)$ which on $[a, b]$ are respectively continuous, continuous and possessing continuous derivatives of the first $n$ orders, (Lebesgue) integrable, (Lebesgue) measurable and $\left|M_{\alpha \beta}(t)\right|^{k}$ integrable, measurable and essentially bounded, a.c., of class $\mathfrak{S}_{p q}^{n-1}[a, b]$ with $M^{[n-1]}(t) \in \mathfrak{N}_{p q}[a, b]$. For brevity, the double subscript $p q$ is reduced to merely $p$ for the $p$-dimensional vector case specified by $p, q=1$, and both subscripts are omitted in the scalar case $p=1, q=1$. For $n \geqq 1$, the subclass of vector functions $y \in \mathfrak{A}_{p}^{n}[a, b]$ for which $y^{[n]}(t) \in \mathfrak{R}_{p}^{2}[a, b]$ is denoted by $\mathfrak{X}_{p}^{n, 2}[a, b]$. Also for $n \geqq 1$ the subclasses of vector functions $y$ belonging to $\mathfrak{C}_{p}^{n}[a, b], \mathfrak{2}_{p}^{n}[a, b], \mathfrak{\Re}_{p}^{n, 2}[a, b]$ for which $y^{[\alpha-1]}(a)=0=y^{[\alpha-1]}(b),(\alpha=1, \cdots, n)$, are denoted by $\mathbb{E}_{p, 0}^{n}[a, b], \mathfrak{2}_{p, 0}^{n}[a, b]$, $\mathfrak{O}_{p, 0}^{n, 2}[a, b]$, respectively. If matrix functions $M(t)$ and $N(t)$ are equal a.e. (almost everywhere) on their interval of definition we write simply $M(t)=N(t)$.

2. Preliminary results. Let $F_{i j}(t)=\left[F_{o \tau ; i j}(t)\right],(i, j=0,1, \cdots, n)$, be $r \times r$ matrix functions defined on an interval $I$ on the real line, and satisfying the following hypothesis.

$F_{n n}(t)$ is nonsingular for $t \in I$, and for arbitrary compact subintervals $[a, b] \subset I$, and $\alpha, \beta=0,1, \cdots, n-1$ we have:

(S)

(a) $F_{n n}, F_{n n}^{-1}, F_{\alpha \beta}, F_{n n}^{-1} F_{n \beta}$ and $F_{\alpha n} F_{n n}^{-1}$ belong to $L_{r r}^{\infty}[a, b]$;

(b) $F_{n \beta}$ and $F_{\alpha n}$ belong to $L_{r r}^{2}[a, b]$.

The $(n+1) r \times(n+1) r$ matrix which for $i, j=0,1, \cdots, n$ and $\sigma, \tau=$ $1, \cdots, r$ has the element in the $(i r+\sigma)$ th row and $(j r+\tau)$ th column equal to $F_{o \tau ; i j}(t)$ will be denoted by $F(t)$, and for $k=0,1, \cdots, n$ the $r \times(n+1) r$ matrix whose element in the $\sigma$ th row and $(j r+\tau)$ th column is $F_{a \tau ; k j}(t)$ will be denoted by merely $F_{k}(t)$. If $[a, b] \subset I$ we shall denote by $\mathfrak{D}[a, b]$ the linear vector space of $r$-dimensional vector functions $y \in \mathfrak{A}_{r}^{n, 2}[a, b]$, and by $\mathfrak{D}_{0}[a, b]$ the subspace consisting of those $y \in \mathfrak{D}[a, b]$ with $y^{[\alpha]}(a)=0=y^{[\alpha]}(b),(\alpha=0,1, \cdots, n-1)$. Also, if $y \in \mathfrak{D}[a, b]$ we shall denote by $\hat{y}$ the $(n+1) r$-dimensional vector function with $\widehat{y}_{j r+\tau}(t)=y_{\tau}^{[j]}(t),(j=0,1, \cdots, n ; \tau=1, \cdots, r)$.

If $[a, b] \subset I$ and $y \in \mathfrak{D}[a, b], z \in \mathfrak{D}[a, b]$ then the integra]

$$
J[y, z \mid a, b]=\int_{a}^{b} \widehat{z}^{*}(t) F(t) \widehat{y}(t) d t
$$

is well defined, and is a sesquilinear form on $\mathfrak{D}[a, b] \times \mathfrak{D}[a, b]$.

LEMMA 2.1. If $y \in \mathfrak{D}[a, b]$, then

$$
J[y, z \mid a, b]=0, \text { for } z \in \mathscr{D}_{0}[a, b]
$$

if and only if $y$ is a solution on $[a, b]$ of the vector quasi-differential 
equation

$$
\mathfrak{R}[y: F](t) \equiv F_{0}(t) \hat{y}(t)-\left\{F_{1}(t) \hat{y}(t)-\left\{\cdots-\left\{F_{n}(t) \hat{y}(t)\right\}^{\prime} \cdots\right\}^{\prime}\right\}^{\prime}=0 .
$$

In conformity with usual terminology, (see, for example, Bradley [2], Reid [9, Sec. 4]), an $r$-dimensional vector function $y(t)$ is a solution of (2.2) if $y \in \mathfrak{D}[a, b]$ and the $r$-dimensional vector functions $v_{k}(t)=\left(v_{o k}(t)\right),(\sigma=1, \cdots, r ; k=1, \cdots, n)$, defined recursively as

$$
\begin{aligned}
& v_{n}(t)=F_{n}(t) \hat{y}(t) \\
& v_{n-p}(t)=F_{n-p}(t) \hat{y}(t)-v_{n-p+1}^{\prime}(t), p=1, \cdots, n-1,
\end{aligned}
$$

all belong to $\mathfrak{U}_{r}[a, b]$ and on $[a, b]$,

$$
\mathscr{L}[y: F](t) \equiv F_{0}(t) \hat{y}(t)-v_{1}^{\prime}(t)=0 .
$$

The result of Lemma 2.1 follows by the classical proof of the fundamental lemma of the calculus of variations, (see, for example, Bliss [1, Sec. 5] for simplest instance; Reid [11, Probs. III. 2:1-8] for more general cases). Indeed, if for an integrable vector function $w(t)$ on $[a, b]$ we introduce $I[w](t)$ for $\int_{a}^{t} w(s) d s$, and for $y \in \mathfrak{D}[a, b]$ we set

$$
\begin{aligned}
& w_{1}(t)=F_{0}(t) \hat{y}(t) \\
& w_{1+p}(t)=F_{p}(t) \hat{y}(t)-I\left[w_{p}\right](t), \quad p=1, \cdots, n-1,
\end{aligned}
$$

then upon suitable integration by parts condition (2.1) becomes

$$
\int_{a}^{b} z^{*[n]}(s)\left\{F_{n}(s) \widehat{y}(s)-I\left[w_{n}\right](s)\right\} d s=0 \text { for } z \in \mathfrak{D}_{0}[a, b] \cdot
$$

By the more familiar form of the fundamental lemma we obtain the existence of a vector polynomial $P_{n-1}(t)$ of degree at most $n-1$ such that on $[a, b]$ we have

$$
F_{n}(t) \hat{y}(t)-I\left[w_{n}\right](t)=P_{n-1}(t) .
$$

Relation (2.7) clearly implies that $v_{n}(t)=I\left[w_{n}\right](t)+P_{n-1}(t)$ is a vector function of class $\mathfrak{U}_{r}[a, b]$ such that $v_{n}=F_{n} \hat{y}$ and

$$
\begin{aligned}
v_{n}^{\prime}(t) & =w_{n}(t)+P_{n-1}^{\prime}(t) \\
& =F_{n-1}(t) \hat{y}(t)-I\left[w_{n-1}\right](t)+P_{n-1}^{\prime}(t) .
\end{aligned}
$$

Then $v_{n-1}(t)=I\left[w_{n-1}\right](t)-P_{n-1}^{\prime}(t)$ is a vector function of class $\mathfrak{A}_{r}[a, b]$ such that $v_{n-1}(t)=F_{n-1}(t) \hat{y}(t)-v_{n}^{\prime}(t)$, and iteration of this procedure leads successively to vector functions $v_{n-p}(t)=I\left[w_{n-p}\right](t)+(-1)^{p} P_{n-1}^{[p]}(t)$ of class $\mathfrak{U}_{r}[a, b]$ and satisfying the equations (2.3). In particular, $v_{1}(t)=I\left[w_{1}\right](t)+(-1)^{n-1} P_{n-1}^{n-1}(t)$ is a vector function of class $\mathfrak{A}_{\mathrm{r}}[a, b]$ satisfying $v_{1}(t)=F_{1}(t) \hat{y}(t)-v_{2}^{\prime}(t)$. Since $P_{n-1}^{[n-1]}(t)$ is constant it then 
follows that $0=w_{1}(t)-v_{1}^{\prime}(t)=F_{0}(t) \hat{y}(t)-v_{1}^{\prime}(t)$, which is the equation (2.2).

Conversely, if $v_{1}(t), \cdots, v_{n}(t)$ are vector functions of class $\mathfrak{A}_{r}[a, b]$ satisfying with a vector function $y \in \mathfrak{D}[a, b]$ the system of equations (2.3), (2.4), then

$$
\begin{aligned}
\hat{z}^{*} F \hat{y} & =z^{*} v_{1}^{\prime}+\sum_{j=1}^{n-1} z^{*[j]}\left[v_{j}+v_{j+1}^{\prime}\right]+z^{*[n]} v_{n} \\
& =\left\{\sum_{\alpha=0}^{n-1} z^{*[\alpha]} v_{\alpha+1}\right\}^{\prime}
\end{aligned}
$$

and consequently (2.1) holds.

For a vector function $y \in \mathfrak{D}[a, b]$, let the $r$-dimensional vector functions $u_{1}(t), \cdots, u_{n}(t)$ be defined as

$$
u_{k}(t)=y^{[k-1]}(t)=\left(u_{\sigma ; k}(t)\right), \quad(k=1, \cdots, n) .
$$

Finally, let $u(t)$ and $v(t)$ denote the $n r$-dimensional vector functions $\left(u_{\rho}(t)\right),\left(v_{\rho}(t)\right),(\rho=1, \cdots, n r)$, with

$$
\begin{aligned}
& u_{i r+\sigma}(t)=y_{o}^{[i]}(t)=u_{o ; i+1}(t), \\
& v_{i r+\sigma}(t)=v_{o ; i+1}(t), \quad(i=0,1, \cdots, n-1 ; \sigma=1, \cdots, r) .
\end{aligned}
$$

The above quasi-differential equation (2.2), or the associated system (2.3), (2.4), may then be written in the matrix form

$$
\begin{aligned}
& \mathscr{L}_{1}[u ; v](t) \equiv-v^{\prime}(t)+C(t) u(t)-D(t) v(t)=0, \\
& \mathscr{L}_{2}[u ; v](t) \equiv u^{\prime}(t)-A(t) u(t)-B(t) v(t)=0,
\end{aligned}
$$

where $A(t), B(t), C(t), D(t)$ are $(n r) \times(n r)$ matrix functions which will be written as partitioned matrices in $r \times r$ matrices as $A(t)=\left[A_{h k}(t)\right]$, $B(t)=\left[B_{h k}(t)\right], C(t)=\left[C_{h k}(t)\right], D(t)=\left[D_{h k}(t)\right],(h, k=1, \cdots, n)$, with

$$
\begin{aligned}
& A_{h k}(t)=\delta_{k, h+1} E_{r},(h=1, \cdots, n-1, k=1, \cdots, n) \\
& \text { (a) } A_{n k}(t)=-F_{n n}^{-1}(t) F_{n, k-1}(t), k=1, \cdots, n ; \\
& \text { (b) } B_{h k}(t)=\delta_{h n} \delta_{n k} F_{n n}^{-1}(t),(h, k=1, \cdots, n) ; \\
& \text { (c) } C_{h k}(t)=F_{h-1, k-1}(t)-F_{h-1, n}(t) F_{n n}^{-1}(t) F_{n, k-1}(t),(h, k=1, \cdots, n) ; \\
& \text { (d) } D_{h k}(t)=\delta_{h, k+1} E_{r},(k=1, \cdots, n-1, h=1, \cdots, n), \\
& D_{h n}(t)=-F_{n-1, n}(t) F_{n n}^{-1}(t),(h=1, \cdots, n) .
\end{aligned}
$$

It is to be noted that whenever hypothesis $(\mathfrak{S})$ is satisfied the differential system (2.10) in $(u ; v)$ is identically normal; that is, if $u(t) \equiv 0, v(t)$ is a solution of $(2.10)$ on a nondegenerate subinterval $I_{0}$ of $I$ then $u(t) \equiv 0, v(t) \equiv 0$ throughout $I$. Indeed, if $u(t) \equiv 0, v(t)$ is a solution of $(2.10)$ on $I_{0}$, then from the equation $\mathscr{L}_{2}[u, v](t)=0$ it follows that $v_{n}(t) \equiv 0$ on $I_{0}$. In turn, from $\mathscr{L}_{1}[u, v](t)=0$ it follows 
that $-v_{h+1}^{\prime}+v_{h}=0,(h=1, \cdots, n-1)$, and consequently also $v_{h}(t) \equiv$ 0 on $I_{0}$ for $h=1, \cdots, n-1$. From the condition $u(t) \equiv 0, v(t) \equiv 0$ on $I_{0}$ it then follows that $u(t) \equiv 0, v(t) \equiv 0$ on $I$, thus establishing the identical normality of $(2.10)$ on $I$.

Two distinct points $t_{1}$ and $t_{2}$ on $I$ are said to be (mutually) conjugate with respect to (2.2), or with respect to (2.10), if there exists a solution $(u(t) ; v(t))$ of this latter system with $u(t) \not \equiv 0$ on the subinterval with endpoints $t_{1}$ and $t_{2}$, while $u\left(t_{1}\right)=0=u\left(t_{2}\right)$. Since $u_{h}(t)=$ $y^{[h-1]}(t),(h=1, \cdots, n)$, this condition states that $t=t_{1}$ and $t=t_{2}$ are zeros of the vector function $y(t)$ of order greater than or equal to $n$. Moreover, if $t_{1} \in I$ and $U(t), V(t)$ are $(n r) \times(n r)$ matrix functions whose column vectors are solutions of (2.10), and satisfying the initial matrix conditions

$$
U\left(t_{1}\right)=0, V\left(t_{1}\right)=E_{n r},
$$

then a value $t_{2} \neq t_{1}$ is conjugate to $t_{1}$ if and only if $U\left(t_{2}\right)$ is singular. If $U\left(t_{2}\right)$ has rank $n r-q$, so that there are $q$ linearly independent solutions $\left(u^{(\rho)}(t) ; v^{(\rho)}(t)\right),(\rho=1, \cdots, q)$, of $(2.10)$ satisfying $u^{(\rho)}\left(t_{1}\right)=$ $0=u^{(\rho)}\left(t_{2}\right)$, then $t_{2}$ is said to be a conjugate point to $t_{1}$ of order $q$.

If $I_{0}$ is a nondegenerate subinterval of $I$ such that no two distinct points of $I_{0}$ are conjugate with respect to (2.2), or (2.10), then this quasi-differential equation or differential system is said to be disconjugate or non-oscillatory on $I_{0}$.

Finally, it is to be noted that $y \in \mathfrak{D}[a, b]$ if and only if the $(n r)-$ dimensional vector function

$$
\begin{array}{r}
\eta(t)=\left(\eta_{\rho}(t)\right), \text { with } \eta_{i r+o}(t)=y_{\sigma}^{[i]}(t), \\
(\sigma=1, \cdots, r ; i=0,1, \cdots, n-1),
\end{array}
$$

has an associated $(n r)$-dimensional vector function $\zeta(t)=\left(\zeta_{\rho}(t)\right) \in \mathbb{R}_{n n}^{2}[a, b]$ such that $\mathscr{L}_{2}[\eta, \zeta](t)=0$ on $[a, b]$. In view of the form of $B(t)$, clearly only the last $r$ components of $\zeta(t)$ are uniquely determined, with values

$$
\zeta_{(n-1) r+\sigma}(t)=\sum_{\tau=1}^{r} F_{\sigma \tau ; n n}(t) y_{\tau}^{[n]}(t),(\sigma=1, \cdots, r) .
$$

3. Self-adjoint systems. The quasi-differential system (2.2), or the equivalent first order system (2.10), is self-adjoint when the coefficient matrix function satisfies in addition to $(\mathfrak{C})$ the further condition

$$
F(t) \text { is hermitian for } t \in I \text {. }
$$

The hermitian character of $F(t)$ is equivalent to the condition that 
the component $r \times r$ matrix functions $F_{i j}$ are such that $\left[F_{i j}(t)\right]^{*}=F_{j i}(t)$ for $t \in I$. In particular, the diagonal component matrix functions $F_{i i}(t)$ are hermitian on $I$. It follows readily that under hypotheses $\left(\mathfrak{S}_{\mathrm{C}}\right)$ and $\left(\mathfrak{S}_{1}\right)$ the coefficient matrices of $(2.10)$ are such that

$$
A(t)=D^{*}(t), B(t)=B^{*}(t), C(t)=C^{*}(t),
$$

and (2.10) is of the canonical form of a linear Hamiltonian system for which one has a generalization of the Sturmian theory for real scalar linear homogeneous differential equations of the second order, (see, in particular, references [5]-[11] of the Bibliography).

Corresponding to the class $\mathfrak{D}[a, b]$ we shall denote by $D[a, b]$ the linear vector space of $(n r)$-dimensional vector functions $\eta(t)$ which are of class $\mathfrak{U}_{n r}[a, b]$, and for which there are corresponding $(n r)$ dimensional vector functions $\zeta(t) \in \mathfrak{R}_{n r}^{2}[a, b]$ such that $\mathscr{L}_{2}[\eta, \zeta](t)=0$ on this interval. The subspace of $D[a, b]$ on which $\eta(a)=0=\eta(b)$ will be denoted by $\boldsymbol{D}_{0}[a, b]$. The fact that a $\zeta(t) \in \mathbb{R}_{n_{r}}^{2}[a, b]$ is thus associated with $\eta(t) \in \mathfrak{N}_{n r}[a, b]$ is denoted by the respective symbols $\eta \in D[a, b]: \zeta$ and $\eta \in D_{0}[a, b]: \zeta$.

When hypotheses $(\mathfrak{K})$ and $\left(\mathfrak{S}_{1}\right)$ hold, and $y^{(p)}(t) \in \mathfrak{D}[a, b],(p=1,2)$, let $\eta^{(p)}(t)=\left(\eta_{\rho}^{(p)}(t)\right),(p=1,2)$, be defined by corresponding equations (2.12), and $\zeta^{(p)}(t)=\left(\zeta_{\rho}^{(p)}(t)\right)$ associated vector functions of class $\mathfrak{R}_{n n}^{2}[a, b]$ whose last $r$ components are specified by equations corresponding to (2.13). The functional $J\left[y^{(1)}, y^{(2)} \mid a, b\right]$ defined by (2.1) is then expressible in terms of $\eta^{(p)}(t), \zeta^{(p)}(t)$ as

$$
J\left[\eta^{(1)}, \eta^{(2)} \mid a, b\right]=\int_{a}^{b}\left\{\zeta^{(2) *} B \zeta^{(1)}+\eta^{(2) * C} \eta^{(1)}\right\} d t,
$$

with the defining relations now equivalent to the condition that $\eta(t)=$ $\eta^{(p)}(t), \zeta(t)=\zeta^{(p)}(t),(p=1,2)$ satisfy the differential equation of restraint

$$
\mathscr{L}_{2}[\eta, \zeta](t)=\eta^{\prime}(t)-A(t) \eta(t)-B(t) \zeta(t)=0 .
$$

As pointed out at the end of the preceding section, if $\eta \in D[a, b]: \zeta$ the vector function $\zeta$ corresponding to a given $\eta$ is not uniquely determined; however, the vector function $B \zeta$ is uniquely determined. Consequently if $\eta^{(p)} \in \boldsymbol{D}[a, b],(p=1,2)$, then the value of the integral in (3.1) is independent of the particular corresponding $\zeta^{(p)}$, so that this integral does indeed define a functional of $\eta^{(1)}, \eta^{(2)}$. Moreover, in view of the hermitian character of the coefficient matrix functions $B$ and $C, J\left[\eta^{(1)}, \eta^{(2)} \mid a, b\right]$ is an hermitian functional on $D[a, b] \times D[a, b]$. In particular, $J[\eta \mid a, b]=J[\eta, \eta \mid a, b]$ given as

$$
J[\eta \mid a, b]=\int_{a}^{b}\left\{\zeta^{*} B \zeta+\eta^{*} C \eta\right\} d t
$$


is a real-valued functional on $D[a, b]$.

For a system (2.10) which satisfies hypotheses $\left(\mathfrak{S}_{2}\right)$ and $\left(\mathfrak{S}_{1}\right)$ it follows readily that if $y^{(p)}=\left(u^{(p)} ; v^{(p)}\right),(p=1,2)$, are solutions of this system then the function

$$
\left(u^{(1)}, v^{(1)} \mid u^{(2)}, v^{(2)}\right)(t)=v_{2}^{*}(t) u_{1}(t)-u_{2}^{*}(t) v_{1}(t)
$$

is constant on $I$. If two solutions of this system are such that this constant is zero, these solutions are said to be (mutually) conjoined. If $Y(t)=(U(t) ; V(t))$ is a $(2 n r) \times q$ matrix whose column vectors are linearly independent solutions of (2.10) which are mutually conjoined, then these solutions form a basis for a conjoined family of solutions of dimension $q$, consisting of these solution of (2.10) which are linear combinations of the column vector functions. In general, (see, for example, Reid [7, Sec. 2; 11, Sec. VII. 2]), the maximal dimension of a conjoined family of solutions of (2.10) is $n r$, and a given conjoined family of dimension less than $n r$ is contained in a conjoined family of dimension $n r$.

If $[a, b]$ is a nondegenerate compact subinterval of $I$, then the symbol $\mathscr{S}_{+}[a, b]$ will signify the condition that the functional $J[y \mid a, b]$ is positive definite on $\mathfrak{D}_{0}[a, b]$; that is, for $y \in \mathfrak{D}_{0}[a, b]$ we have $J[y \mid a, b] \geqq 0$, with the equality sign holding only if $y(t)=0$ on $[a, b]$. This condition may be equally well stated as the nonnegativeness of the functional (3.3) on the vector space $D_{0}[a, b]$, with $J[\eta \mid a, b]=0$ for an $\eta \in D_{0}[a, b]: \zeta$ only if $\eta(t)=0$ and $B(t) \zeta(t)=0$ on $[a, b]$.

From the basic result for canonical Hamiltonian systems concerning disconjugacy on a compact interval, (see, for example, Reid [10, Theorem 5.1] or Reid [11, Sec. VII. 4]), we have the following criterion.

THEOREM 3.1. If hypotheses $\left(\mathfrak{S}_{2}\right)$ and $\left(\mathfrak{F}_{1}\right)$ are satisfied, and $[a, b]$ is a nondegenerate compact subinterval of $I$, then $\mathfrak{F}_{+}[a, b]$ holds if and only if $F_{n n}(t)>0$ for $t$ a.e. on $[a, b]$, together with one of the following conditions:

(i) (2.10) is disconjugate on $[a, b]$;

(ii) there exists a conjoined family of solutions $Y(t)=(U(t) ; V(t))$ of (2.10) of dimension $n r$ with $U(t)$ nonsingular on $[a, b]$.

4. A disconjugacy criterion for (2.2). Suppose that hypothesis $(\mathfrak{S})$ is satisfied by the coefficient matrix function $F(t)$ of $(2.2)$ on an interval $I$, and that $[a, b]$ is a nondegenerate subinterval of $I$ such that $t=a$ and $t=b$ are mutually conjugate with respect to the equation (2.2). Let $y(t)$ be a solution of (2.2) such that $y(t) \not \equiv 0$ on $[a, b]$, and $y^{[\alpha]}(a)=0=y^{[\alpha]}(b),(\alpha=0,1, \cdots, n-1)$. Then $y \in \mathfrak{D}_{0}[a, b]$, and in view of Lemma 2.1 we have that 


$$
0=J[y, y \mid a, b]=\int_{a}^{b} \hat{y}^{*}(t) F(t) \hat{y}(t) d t .
$$

From this relation it follows that $\mathfrak{R e} F(t)=: \frac{1}{2}\left\{F(t)+F^{*}(t)\right\}$ and $\mathfrak{m} F(t)=$ $\frac{1}{2} \sqrt{-1}\left\{F^{*}(t)-F(t)\right\}$ are hermitian matrix functions. If $\lambda_{0}, \lambda_{1}$ are real constants then

$$
F(t ; \lambda)=\lambda_{0} \Re \mathrm{Re}(t)+\lambda_{1} \mathfrak{\Im} \boldsymbol{m} F(t)
$$

is an hermitian matrix function such that the given solution $y(t)$ of (2.2) satisfies the condition

$$
\int_{a}^{b} \hat{y}^{*}(t) F(t ; \lambda) \hat{y}(t) d t=0 .
$$

Now if $F(t ; \lambda)$ has the partitioned representation $\left[F_{i j}(t ; \lambda)\right],(i, j=$ $0,1, \cdots, n)$ in terms of $r \times r$ matrix functions, and $F(t ; \lambda)$ satisfies hypothesis $(\mathfrak{S})$ with $F_{n n}(t ; \lambda)>0$ for $t$ a.e. on $[a, b]$, then the conclusion (i) of Theorem 3.1 applied to the self-adjoint matrix differential equation $2[y: F(\cdot ; \lambda)](t)=0$ implies that this equation fails to be disconjugate on $[a, b]$. Consequently, we have the following result, corresponding to that of $\S 5$ of Reid [7] for a second order linear homogeneous matrix differential equation. The reader is also referred to Hartman and Wintner [3] for a similar treatment of disconjugacy criteria for second order vector differential systems. For a consideration of non-self-adjoint differential equations of even order by a method which is similar in basic idea, but different in specific detail, see Kreith [4].

THEOREM 4.1. Suppose that hypothesis (F) is satisfied by the coefficient matrix function $F(t)$ of (2.2) on an interval $I$, and for a given nondegenerate subinterval $[a, b]$ of $I$ there exist real constants $\lambda_{0}, \lambda_{1}$ such that on $[a, b]$ the matrix function $F(t ; \lambda)=\left[F_{i j}(t ; \lambda)\right],(i, j=$ $0,1, \cdots, n)$, of $(4.2)$ satisfies hypothesis $(\mathfrak{S})$ and $F_{n n}(t ; \lambda)>0$ for $t$ a.e. on $[a, b]$. Then whenever the self-adjoint quasi-differential equation $\Omega[y: F(\cdot ; \lambda)](t)=0$ is disconjugate on $[a, b]$, the system (2.2) is also disconjugate on $[a, b]$.

It is to be emphasized that in the above theorem the constant multipliers $\lambda_{0}, \lambda_{1}$ may depend upon the subinterval $[a, b]$, and that any criterion of disconjugacy for the associated self-adjoint equation 2. $[y: F(\cdot ; \lambda)](t)=0$ yields a sufficient condition for disconjugacy of the original equation (2.2). In particular, the results of Reid [9, Sec. 4] for scalar quasi-differential equations of even order, and their analogues for vector equations, provide sufficient conditions for (2.2) to be disconjugate on a non-compact interval $\left(t_{1}, \infty\right)$. 
5. A special canonical form. Attention will be directed now to a linear differential expression of order $m$ in the $r$-dimensional vector function $y(t)=\left(y_{\sigma}(t)\right)$ of the form

$$
\mathscr{L}[y](t)=\sum_{\mu=0}^{m} P_{\mu}(t) y^{[\mu]}(t)
$$

where the $r \times r$ coefficient matrix functions $P_{\mu}(t) \equiv\left[P_{\sigma \tau ; \mu}(t)\right]$ are supposed to be of class $\mathfrak{R}_{r r}[a, b]$ for arbitrary compact subintervals $[a, b]$ of a given interval $I$ on the real line. It is to be emphasized that in the discussion leading to the result of Theorem 5.1 we do not require the leading coefficient matrix $P_{m}(t)$ to be nonsingular, or even to be nonzero. The purpose of this section is to present for vector differential operators of the form (5.1) an analogue of the results of Reid [8] for linear scalar differential equations, and to note the particular form of the disconjugacy criterion of $\S 4$ for the involved canonical form.

For a given compact subinterval $[a, b]$ of $I$, let $T_{0}$ denote a corresponding differential operator with domain $\left.\left.\mathbb{C}_{r, 0}^{m}\right] a, b\right]$ and value $T_{0} y=\mathscr{L}[y]$. If $\mathfrak{D}^{*}$ denotes the totality of $r$-dimensional vector functions $z \in \mathfrak{R}_{r r}[a, b]$ with $P_{\mu}^{*}(t) z(t) \in \mathfrak{R}_{r r}[a, b],(\mu=0,1, \cdots, m)$, and for which there exists a corresponding $f_{z} \in \mathfrak{R}_{r}[a, b]$ such that

$$
\int_{a}^{b} z^{*} \mathscr{L}[y] d t=\int_{a}^{b} f_{z}^{*} y d t, \text { for } y \in C_{r, 0}^{m}[a, b],
$$

then the operator $T_{0}^{*}$ with domain $\mathfrak{D}^{*}$ and value $T_{0}^{*} z=f_{z}$ is termed the adjoint of $T_{0}$. In particular, if $P_{\mu} \in \mathfrak{S}_{r r}^{\mu}[a, b]$ and $P_{m}(t)$ is nonsingular for $t \in[a, b]$, then by classical results, (see, for example, Reid [11, Sec. III. 9]) we have that $\mathfrak{D}^{*}=\mathfrak{A}_{r}^{m}[a, b]$, and for $z \in \mathfrak{A}_{r}^{m}[a, b]$ the value of $T_{0}^{*} z$ is given by the Lagrange adjoint $\sum_{\mu=0}^{m}(-1)^{\mu}\left\{P^{*} z\right\}^{[\mu]}$. Of special importance is the Hilbert space case that occurs when $P_{\mu} \in \mathbb{R}_{r r}^{2}[a, b]$, $(\mu=0,1, \cdots, m)$, and analogous to the above defined $T_{0}$ one considers the operator with values $\mathscr{L}[y]$ on the domain of functions $y \in \mathfrak{Q}_{r, 0}^{m}[a, b]$ such that $\mathscr{L}[y] \in \mathbb{R}_{r}^{2}[a, b]$.

Of particular significance for the present considerations are differential expressions $\mathscr{L}[y]=\Lambda_{q}[y ; P]$ where $P$ is an $r \times r$ matrix function, and

$$
\begin{aligned}
& \Lambda_{0}[y ; P](t)=P(t) y(t), \Lambda_{2 p}[y ; P](t)=\left\{P(t) y^{[p]}(t)\right\}^{[p]}, \\
& \Lambda_{2 p-1}[y ; P](t)=\left\{P(t) y^{[p-1]}(t)\right\}^{[p]}+\left\{P(t) y^{[p]}(t)\right\}^{[p-1]}, \quad(p=1,2, \cdots),
\end{aligned}
$$

with the understanding that in the definition of $\Lambda_{2 p}$ and $\Lambda_{2 p-1}$ the involved matrix function $P$ is of class $\mathfrak{A}_{r}^{p}[a, b]$. If for (5.1) we have $\mathscr{L}[y]=\Lambda_{m}[y ; P],(m \geqq 1)$, then the fact that $\mathfrak{2}_{r}^{m}[a, b] \subset \mathfrak{D}^{*}$ and $T_{0}^{*} z=$ $\Lambda_{m}\left[z ;(-1)^{m} P^{*}\right]$ for $z \in \mathfrak{N}_{r}^{m}[a, b]$ is a direct consequence of the well- 
known equation

$$
z^{*} \Lambda_{m}[y ; P]-(-1)^{m}\left\{\Lambda_{m}\left[z ; P^{*}\right]\right\}^{*} y=\left\{K_{n}[y, z ; P]\right\}^{\prime}
$$

for arbitrary $y, z$ of $\mathfrak{A}_{r}^{m}[a, b]$, where $K_{n}[y, z ; P]$ is the so-called bilinear concomitant of the form $\sum_{\mu, \nu=1}^{m} z^{*[\nu-1]}(t) K_{\nu \mu}(t ; P) y^{[\mu-1]}(t)$.

Let $e^{(k)}$ denote the $r$-dimensional unit vector $e^{(k)}=\left(\delta_{h k}\right),(h=$ $1, \cdots, r)$, and designate by $g_{\lambda}(t),(\lambda=0,1, \cdots)$ the particular scalar polynomials $g_{0}(t) \equiv 1, g_{\lambda}(t)=t^{\lambda} / \lambda !, \quad(\lambda=1,2, \cdots)$. Moreover, let $k_{j}$ equal $j / 2$ or $(j+1) / 2$ according as $j$ is even or odd. Corresponding to Theorem 3.2 of Reid [8], we now have the following representation theorem.

THEOREM 5.1. Suppose that $\mathscr{L}[y]$ is given by (5.1) with $P_{\mu} \in \mathbb{R}_{r r}[a, b]$, $(\mu=0,1, \cdots, m)$, and the differential operator $T_{0}$ is defined as specified above. If for $h=1, \cdots, r$ and $\lambda=0,1, \cdots, k_{m}-1$ the vector functions $g_{\lambda}(t) e^{(h)}$ belong to $\mathfrak{D}^{*}$, then there exist matrix functions $\Pi_{\mu}(t) \in \mathfrak{A}_{r}^{k}[a, b],(\mu=0,1, \cdots, m)$, such that

$$
\mathscr{L}[y](t)=\sum_{\mu=0}^{m} \Lambda_{\mu}\left[y ; \Pi_{\mu}\right](t) \text { for } y \in \mathfrak{U}_{r}^{m}[a, b] ;
$$

also $\mathfrak{A}_{r}^{m}[a, b] \subset \mathfrak{D}^{*}$ and

$$
\left(T_{0}^{*} z\right)(t)=\mathscr{L}^{*}[z](t)=\sum_{\mu=0}^{m} \Lambda_{\mu}\left[z ;(-1)^{\mu} \Pi_{\mu}^{*}\right](t), \text { for } z \in \mathfrak{P}_{r}^{m}[a, b] .
$$

Moreover, $\Pi_{\mu} \in \mathfrak{P}_{r}^{k_{\mu}, 2}[a, b],(\mu=0,1, \cdots, m)$, if and only if

$$
T_{0}^{*}\left\{g_{\lambda} e^{(h)}\right\} \in \mathfrak{R}_{r}^{2}[a, b],\left(h=1, \cdots, r ; \lambda=0,1, \cdots, k_{m}-1\right),
$$

and $P_{\mu} \in \mathbb{R}_{r r}^{2}[a, b],\left(\mu=0,1, \cdots, m-k_{m}\right)$.

The result of the above theorem is a direct consequence of Theorem 3.2 of Reid [8] applied to the associated scalar differential operators

$$
\mathscr{L}_{h k}[u](t)=\sum_{\mu=0}^{m}\left\{e^{(h) *} P_{\mu}(t) e^{(k)}\right\} u^{[\mu]},(h, k=1, \cdots, r),
$$

and expressing in matrix form the scalar results thus obtained.

If for a differential expression (5.1) with $m=2 n$ we have that $\mathscr{L}[y]$ is given in a corresponding form (5.4) then the differential equation $\mathscr{L}[y](t)=0$ is of the form (2.2) with the $(n+1) r \times(n+1) r$ matrix function $F(t)$ expressible in partitioned form $\left[F_{i j}(t)\right]$ with $F_{i j}$, $(i, j=0,1, \cdots, n)$, the $r \times r$ matrix functions specified for $i, j=$ $0,1, \cdots, n$ as 


$$
\begin{aligned}
& F_{i j}(t)=0, \text { if }|i-j|>1 ; \\
& F_{i j}(t)=(-1)^{i} \Pi_{i+j}(t), \text { if }|i-j| \leqq 1
\end{aligned}
$$

For such a matrix function $F(t)$ we have that $\Re$ e $F(t)=G(t) \equiv\left[G_{j k}(t)\right]$, $(j, k=0,1, \cdots, n)$, where each $G_{j k}$ is an $r \times r$ matrix function specified for $j, k=0,1, \cdots, n$ as

$$
\begin{aligned}
G_{j k}(t) & =0, \text { if }|j-k|>1 ; \\
G_{j j}(t) & =(-1)^{j} \Re e \Pi_{2 j}(t) ; \\
G_{j, j+1}(t) & =\sqrt{-1}(-1)^{j} \Im \mathfrak{m} \Pi_{2 j+1}(t) ; \\
G_{j, j-1}(t) & =\sqrt{-1}(-1)^{j} \Im \mathfrak{m} \Pi_{2 j-1}(t) .
\end{aligned}
$$

Correspondingly, $\Im_{\mathfrak{m}} F(t)=H(t)=\left[H_{j k}(t)\right],(j, k=0,1, \cdots, n)$, where each $H_{j k}$ is an $r \times r$ matrix function specified for $j, k=0,1, \cdots, n$ as

$$
\begin{aligned}
H_{j k}(t) & =0, \text { if }|j-k|>1 ; \\
H_{j j}(t) & =(-1)^{j} \mathfrak{J} \Pi_{2 j}(t) ; \\
H_{j, j+1}(t) & =\sqrt{-1}(-1)^{j+1} \Re e \Pi_{2 j+1}(t) ; \\
H_{j, j-1}(t) & =\sqrt{-1}(-1)^{j+1} \Re \mathrm{e} \Pi_{2 j-1}(t) .
\end{aligned}
$$

As an application of the result of Theorem 4.1 with multipliers $\lambda_{0}=1, \lambda_{1}=0$, or $\lambda_{0}=-1, \lambda_{1}=0$, one has the following special criterion for disconjugacy of a differential equation (2.2).

THEOREM 5.2. Suppose that (5.1) with $m=2 n$ is expressible in the form (5.4) with coefficient matrices $\Pi_{0}(t), \cdots, \Pi_{2 n}(t)$ satisfying the conditions given in Theorem 5.1, while $\mathfrak{I}_{\mathfrak{m}} \Pi_{2 j-1}(t)=0, j=1, \cdots, n$, and on a given nondegenerate compact subinterval $[a, b]$ of $I$ we have either $\Re \Pi_{2 n}(t)>0$ or $\Re \Pi_{2 n}(t)<0$. If the associated self-adjoint differential system

$$
\mathscr{L}_{1}[y](t)=\sum_{j=0}^{n} \Lambda_{2 j}\left[y ; \Re e \Pi_{2 j}\right](t)=0
$$

is disconjugate on $[a, b]$ then the differential equation (5.4) is also disconjugate on this subinterval.

In particular, the functions $\mathfrak{I} m \Pi_{2 j-1}(t),(j=1, \cdots, n)$ are all zero in the scalar case when $r=1$, and the coefficients of (5.1) are realvalued.

\section{REFERENCES}

1. G. A. Bliss, Lectures on the Calculus of Variations, The University of Chicago Press, 1946.

2. J. S. Bradley, Adjoint quasi-differential operators of Euler type, Pacific J. Math. 
16 (1966), 213-237.

3. P. Hartman, and A. Wintner, On disconjugate differential systems, Canad. J. Math. 8 (1956), 72-81.

4. K. Kreith, Disconjugacy criteria for non-selfadjoint differential equations of even order, Canad. J. Math., 23 (1971), 644-652.

5. M. Morse, Sufficient conditions in the problem of Lagrange with variable end conditions, Amer. J. Math. 53 (1931), 517-546.

6 . The Calculus of Variations in the Large, American Mathematical Society Colloquium Publications, XVIII (1934).

7. W. T. Reid, Oscillation criteria for linear differential systems with complex coefficients, Pacific J. Math., 6 (1956), 733-751.

8. - Adjoint linear differential equations, Trans. Amer. Math. Soc., 85 (1957), 446-461.

9. — Oscillation criteria for self-adjoint differential systems. Trans. Amer. Math. Soc., 101 (1961), 91-106.

10. - Principal solutions of nonoscillatory linear differential systems, J. Math. Anal. Appl. 9 (1964), 397-423.

11. — Ordinary Differential Equations, John Wiley and Sons, 1971.

Received November 4, 1970. This research was supported by the Air Force Office of Scientific Research, Office of Aerospace Research, United States Air Force, under Grant AFOSR-68-1398B. The United States Government is authorized to reproduce and distribute reprints for governmental purposes notwithstanding any copyright notation hereon.

THE UNIVERSITY OF OKLAHOMA 


\title{
PACIFIC JOURNAL OF MATHEMATICS
}

\author{
EDITORS
}

\author{
H. SAMELSON \\ Stanford University \\ Stanford, California 94305 \\ C. R. HOBBY \\ University of Washington \\ Seattle, Washington 98105
}

J. DugundJI

Department of Mathematics

University of Southern California

Los Angeles, California 90007

RICHARD ARENS

University of California

Los Angeles, California 90024

\section{ASSOCIATE EDITORS}
E. F. BeCKenBaCH
B. H. NeUMANN
F. WOLF
K. Yoshida

\section{SUPPORTING INSTITUTIONS}

\author{
UNIVERSITY OF BRITISH COLUMBIA \\ CALIFORNIA INSTITUTE OF TECHNOLOGY \\ UNIVERSITY OF CALIFORNIA \\ MONTANA STATE UNIVERSITY \\ UNIVERSITY OF NEVADA \\ NEW MEXICO STATE UNIVERSITY \\ OREGON STATE UNIVERSITY \\ UNIVERSITY OF OREGON \\ OSAKA UNIVERSITY
}

\author{
UNIVERSITY OF SOUTHERN CALIFORNIA \\ STANFORD UNIVERSITY \\ UNIVERSITY OF TOKYO \\ UNIVERSITY OF UTAH \\ WASHINGTON STATE UNIVERSITY \\ UNIVERSITY OF WASHINGTON \\ AMERICAN MATHEMATICAL SOCIETY \\ NAVAL WEAPONS CENTER
}

The Supporting Institutions listed above contribute to the cost of publication of this Journal, but they are not owners or publishers and have no responsibility for its content or policies.

Mathematical papers intended for publication in the Pacific Journal of Mathematics should be in typed form or offset-reproduced, (not dittoed), double spaced with large margins. Underline Greek letters in red, German in green, and script in blue. The first paragraph or two must be capable of being used separately as a synopsis of the entire paper. The editorial "we" must not be used in the synopsis, and items of the bibliography should not be cited there unless absolutely necessary, in which case they must be identified by author and Journal, rather than by item number. Manuscripts, in duplicate if possible, may be sent to any one of the four editors. Please classify according to the scheme of Math. Rev. Index to Vol. 39. All other communications to the editors should be addressed to the managing editor, Richard Arens, University of California, Los Angeles, California, 90024.

50 reprints are provided free for each article; additional copies may be obtained at cost in multiples of 50 .

The Pacific Journal of Mathematics is published monthly. Effective with Volume 16 the price per volume (3 numbers) is $\$ 8.00$; single issues, $\$ 3.00$. Special price for current issues to individual faculty members of supporting institutions and to individual members of the American Mathematical Society: $\$ 4.00$ per volume; single issues $\$ 1.50$. Back numbers are available.

Subscriptions, orders for back numbers, and changes of address should be sent to Pacific Journal of Mathematics, 103 Highland Boulevard, Berkeley, California, 94708.

PUBLISHED BY PACIFIC JOURNAL OF MATHEMATICS, A NON-PROFIT CORPORATION

Printed at Kokusai Bunken Insatsusha (International Academic Printing Co., Ltd.), 270, 3chome Totsuka-cho, Shinjuku-ku, Tokyo 160, Japan. 


\section{Pacific Journal of Mathematics}

\section{Vol. 39, No. $3 \quad$ July, 1971}

William O'Bannon Alltop, 5-designs in affine spaces ................... 547

B. G. Basmaji, Real-valued characters of metacyclic groups ................. 553

Miroslav Benda, On saturated reduced products....................... 557

J. T. Borrego, Haskell Cohen and Esmond Ernest Devun, Uniquely representable semigroups. II.......................................

George Lee Cain Jr. and Mohammed Zuhair Zaki Nashed, Fixed points and stability for a sum of two operators in locally convex spaces ....................

Donald Richard Chalice, Restrictions of Banach function spaces ...............

Eugene Frank Cornelius, Jr., A generalization of separable groups ..............

Joel L. Cunningham, Primes in products of rings ......................

Robert Alan Morris, On the Brauer group of $Z$.

593

603

615

David Earl Dobbs, Amitsur cohomology of algebraic number rings ...............

Charles F. Dunkl and Donald Edward Ramirez, Fourier-Stieltjes transforms and

weakly almost periodic functionals for compact groups ...................

Hicham Fakhoury, Structures uniformes faibles sur une classe de cônes et

d'ensembles convexes ......................................

Leslie R. Fletcher, A note on C $\theta \theta$-groups.

Humphrey Sek-Ching Fong and Louis Sucheston, On the ratio ergodic theorem for

semi-groups............................................

James Arthur Gerhard, Subdirectly irreducible idempotent semigroups ...........

Thomas Eric Hall, Orthodox semigroups.....................

Marcel Herzog, $C \theta \theta$-groups involving no Suzuki groups ..........

669

687

John Walter Hinrichsen, Concerning web-like continua ..........

691

Frank Norris Huggins, A generalization of a theorem of F. Riesz.

695

Carlos Johnson, Jr., On certain poset and semilattice homomorphisms

703

Alan Leslie Lambert, Strictly cyclic operator algebras ...........

717

Howard Wilson Lambert, Planar surfaces in knot manifolds . . .

727

Robert Allen McCoy, Groups of homeomorphisms of normed linear spaces ....... 735

T. S. Nanjundiah, Refinements of Wallis's estimate and their generalizations ...... 745

Roger David Nussbaum, A geometric approach to the fixed point index .......... 751

John Emanuel de Pillis, Convexity properties of a generalized numerical range .... 767

Donald C. Ramsey, Generating monomials for finite semigroups ....

783

William T. Reid, A disconjugacy criterion for higher order linear vector differential equations...

Roger Allen Wiegand, Modules over universal regular rings...

Kung-Wei Yang, Compact functors in categories of non-archimedean Banach

spaces.

R. Grant Woods, Correction to: "Co-absolutes of remainders of Stone-Čech compactifications".

Ronald Owen Fulp, Correction to: "Tensor and torsion products of

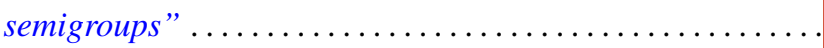

Bruce Alan Barnes, Correction to: "Banach algebras which are ideals in a banach 